Short communication

\title{
Minimal clinically important difference for the historic parts of the Unified Dyskinesia Rating Scale
}

\author{
Attila Makkos ${ }^{\mathrm{a}, \mathrm{b}}$, Márton Kovács ${ }^{\mathrm{a}, \mathrm{b}}$, Dávid Pintér ${ }^{\mathrm{a}}$, József Janszky ${ }^{\mathrm{b}, \mathrm{c}}$, Norbert Kovács ${ }^{\mathrm{b}, \mathrm{c}, *}$ \\ ${ }^{\text {a }}$ Doctoral School of Clinical Neuroscience, University of Pécs, Pécs, Hungary \\ ${ }^{\mathrm{b}}$ Department of Neurology, University of Pécs, Pécs, Hungary \\ ${ }^{\mathrm{c}}$ MTA-PTE Clinical Neuroscience MR Research Group, Pécs, Hungary
}

\section{A R T I C L E I N F O}

\section{Keywords:}

Minimal clinically important changes Minimal but clinically relevant differences Receiver operating characteristic curve Unified Dyskinesia Rating Scale Parkinson disease

\begin{abstract}
A B S T R A C T
Background: Motor complications represent an important clinical problem in the treatment of Parkinson's disease (PD). The Motor Complications Part of the Movement Disorder Society-sponsored Unified Parkinson's Disease Rating Scale (MDS-UPDRS Part IV) and the Unified Dyskinesia Rating Scale (UDysRS) are among the most reliable instruments to evaluate these problems. The minimal clinically important difference thresholds are the smallest changes in the outcome measures that are clinically meaningful.

Aims: The aim of our study was to calculate the minimal clinically important difference thresholds for the MDSUPDRS Part IV and the historic parts of the UDysRS.

Methods: A total of 1044 paired investigations of 436 patients were analyzed. Changes in the respective outcome measures (MDS-UPDRS Part IV, UDysRS Parts I and II) were compared to the Patient-rated Global Impression of Improvement scores (anchors). Subsequently, we applied receiver-operating characteristic analysis to ascertain the MCID thresholds with optimal sensitivity and specificity.

Results: Any improvement greater than 2.1 points or any worsening greater than 1.8 points on UDysRS Part I represents a minimal, yet clinically meaningful change. In reference to UDysRS Part II, the smallest changes considered clinically relevant are 1.8 and 1.7 points for improvement and deterioration, respectively. The thresholds for the MDS-UPDRS Part IV are 0.9 points for improvement and 0.8 points for worsening.

Conclusions: Our estimates may allow the judgment of the clinical relevance of numeric changes in the dyskinesia scales.
\end{abstract}

\section{Introduction}

Parkinson's disease (PD) is the second most frequent neurodegenerative disorder. Current therapeutic approaches have mainly systematic effects on the motor and non-motor symptoms of the disorder. As the disease progresses, the therapeutic efficacy of antiparkinsonian medication becomes more and more limited, and motor complications inevitably develop. Wearing off, on-off fluctuations, peak of dose dyskinesia, biphasic dyskinesia, and mainly OFF state dystonia are the most common long-term problems of the dopaminergic drugs. Many therapeutic approaches, including oral combinations, deep brain stimulation (DBS), levodopa/carbidopa intestinal gel infusion (LCIG), and apomorphine pump treatments, aim to improve the symptoms of $\mathrm{PD}$ and reduce the motor and non-motor complications simultaneously. A growing number of interventional trials aimed at reducing the motor complications have been published recently. To judge their efficacy, valid and reliable clinical outcome measures are warranted.

Recently a single, comprehensive rating scale, called the Unified Dyskinesia Rating Scale (UDysRS), was developed to assess the most important dimensions of motor complications [1]. The development of the scale was mainly clinimetrically driven [2] to simultaneously and reliably measure various aspects of the motor complications [3]. The

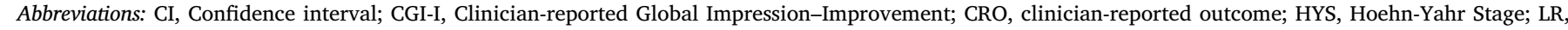

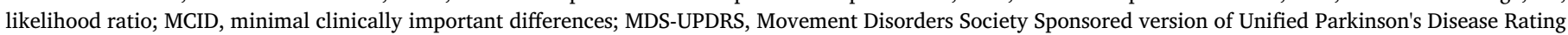

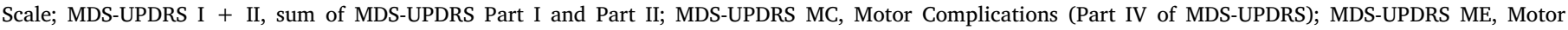

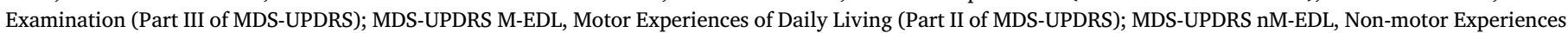

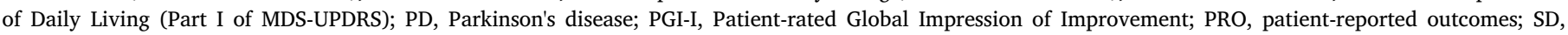
standard deviation; UDysRS, Unified Dyskinesia Rating Scale

* Corresponding author. Department of Neurology, University of Pécs, 7633, Pécs, Rét utca 2, Hungary.

E-mail address: kovacs.norbert@pte.hu (N. Kovács). 
UDysRS consists of four different subscales representing different domains:

- Part 1 ON Dyskinesia measures the subjective impact of ON dyskinesia on everyday activities. The first item on the time spent with ON dyskinesia is assessed by a trained rater (Part $1 \mathrm{~A}$ ), whereas the remaining 10 items (Part $1 \mathrm{~B}$ ) are self-rated by patients.

- Part 2 OFF Dystonia evaluates the burden caused by OFF dystonia. The first item on the time spent with OFF dystonia is evaluated by a trained rater (Part $2 \mathrm{~A}$ ), and the remaining three items (Part $2 \mathrm{~B}$ ) are completed by patients.

- Part 3 Impairment assesses the tropical distribution and severity of dyskinesia.

- Part 4 Disability examines the disability associated with dyskinesia on four representative tasks, including communication, drinking, dressing, and ambulation.

With the exception of the first questions assessing the time spent with ON dyskinesia and OFF dystonia, Parts 1 and 2 form a self-rated questionnaire, a type of patient-reported outcome (PRO) commonly referred as the "historic" sections of the UDysRS. Parts 3 and 4 are assessed by clinicians (clinician-rated outcome, CRO) usually denoted as "objective" parts of the UDysRS [1]. While the individual subscales can be evaluated independently, the total score of the UDysRS can also be calculated by summing the scores on the four domains [1]. Recent studies have demonstrated that the UDysRS simultaneously has a good temporal stability [4] and a reliable responsiveness measuring changes in motor complications [5]. Moreover, the UDysRS can also be used reliably over the Web [6], making it a feasible instrument for patients unable to physically attend their physician's office. Another advantage of the scale is the development of numerous official language versions [7-9] in accordance with the strict rules for validation [2].

In addition to UDysRS, the Motor Complications part of the Movement Disorder Society-sponsored Unified Parkinson's Disease Rating Scale (MDS-UPDRS MC, Part IV) [10] is also capable of measuring the motor complications in a simple, less detailed and comprehensive, yet commonly reported, way.

In biomedical research, statistical significance does not necessarily imply clinical relevance. Because small changes in the outcome measures do not unequivocally have clinical meaning, the concept of the minimal clinically important difference (MCID) has been introduced. The MCID represents the smallest changes in an outcome that the patient acknowledges as meaningful. At this time, only limited data are available on the MCID thresholds of the dyskinesia rating scales. Although Mestre et al. [11] demonstrated that a 2.32-point change on the clinician-rated UDysRS Part 3 is the threshold for clinical relevance, the MCID estimates for the historic parts of the UDysRS have not yet been calculated. Although our team evaluated the MCID thresholds for Parts I [12], II [12], III [13], and the total score [14] of the MDSUPDRS, the size of the minimally required change on the MDS-UPDRS MC is still unknown.

Fulfilling this clinical need, we aimed to evaluate the MCID thresholds for the recently published dyskinesia scales, the MDS-UPDRS $\mathrm{MC}$ and the historic parts of the UDysRS.

\section{Materials and methods}

The study protocol was similar to the procedure of measuring the MCID thresholds for the motor and non-motor experiences of daily living parts of the MDS-UPDRS [12]. A total of 436 patients with PD and motor complications at baseline were enrolled and sequentially examined every 6 months. The study was approved by the Regional and Institutional Ethical Committee (3617.316-24987/KK41). Besides demographic-, medication-, and disease-related data, the validated Hungarian versions of the MDS-UPDRS [15] and UDysRS [8] were assessed. At follow-up visits, both the Clinician-reported Global Impression of
Improvement (CGI-I) and the Patient-rated Global Impression of Improvement (PGI-I) [16] scales were obtained to measure the perceived changes in the motor complications since the last examination. Subjects with the major neurocognitive disorder were excluded (Montreal Cognitive Assessment score < 20.5) [17], [18]. The severity of PD was categorized based on the Hoehn-Yahr Stage (HYS) as mild (HYS 1 and 2), moderate (HYS 3), and severe (HYS 4 and 5) [19].

Following the recommendations of Revicki et al. [20], both anchorand distribution-based methods were used for the MCID calculations [20]. Clinimetrically, the anchor for MCID calculations should be an independent and clinically relevant instrument that is simultaneously interpretable by itself and correlates with the outcome measure being evaluated $[20,21]$. First, we tested whether the CGI-I or PGI-I is feasible by calculating the Spearman correlation coefficients between the changes in dyskinesia scores and the potential anchors. As a minimum of 0.3 is required for the correlation coefficient [20], the PGI-I was chosen for further analyses (Supplementary data). We performed ordinal regression modeling between the PGI-I (dependent value) and the changes in the evaluated dyskinesia scores to verify its feasibility as an anchor [12]. The detailed methods for MCID threshold calculations were published previously [13]. First, we compared the changes in dyskinesia measures with the PGI-I score 4 (no change) with the changes in the dyskinesia scales associated with PGI-I score 3 (minimal improvement) and PGI-I score 5 (minimal worsening). Subsequently, we applied the receiver-operating characteristic (ROC) technique to identify the optimal threshold values with the highest sensitivity and specificity. Finally, effect size calculations (Cohen d) were performed [22]. In optimal cases, both anchor-based calculations should yield similar MCID values [20,23], representing an approximately 0.2 effect size $[20,22]$.

\section{Results}

The 1044 paired visits of 436 patients with PD were analyzed. The median number of return visits was 2 . The baseline characteristics of the patients and the changes in the antiparkinsonian treatment are displayed as Supplementary data. We could build statistically significant ordinal regression models between the PGI-I and the changes in the dyskinesia scales (Nagelkerke pseudo-R-square values: 0.378, 0.397, and 0.328 for UDysRS Part 1, UDysRS Part 2, and MDS-UPDRS $\mathrm{MC}$, respectively; $\mathrm{p}<0.05$ in all instances).

The mean change in the dyskinesia scores in the group of patients having a PGI-I score of 4 was negligible, with the effect size varying between 0.01 and 0.04 . Table 1 shows the mean changes, the effect size, and the specificity and sensitivity values denoting the full dataset; the MCID estimates for the different severity stages are shown in Table 2.

\section{Discussion}

Although an increasing number studies use the UDysRS Parts 1 and 2 and the MDS-UPDRS MC as their outcome measures, the MCID thresholds for these instruments had not yet been evaluated. Therefore, the results of these studies could only be judged from the statistical point of view. Following the recommendations of Revicki et al. [20], we aimed to calculate these MCID thresholds, providing a judgment for further clinical practice. We demonstrated that any improvement greater than 2.1 points or any worsening greater than 1.8 points on UDysRS Part 1 represents a minimal, yet clinically meaningful change. In reference to UDysRS Part 2, the smallest changes considered clinically relevant are 1.8 and 1.7 points for improvement and deterioration, respectively. The thresholds for the MDS-UPDRS MC are 0.9 points for improvement and 0.8 points for worsening.

The strength of our approach was the simultaneous use of various anchor- and distribution-based methods giving similar MCID estimations. The relatively large number of enrolled patients and a wide range of disease severity examined can further ensure the reliability and 
Table 1

Mean changes in certain UDysRS and MDS-UPDRS scores compared to Patient-rated Global Impression of Improvement scores.

\begin{tabular}{|c|c|c|c|c|c|c|c|c|c|c|c|c|c|}
\hline \multirow[t]{2}{*}{ Instrument } & \multicolumn{2}{|c|}{ PGI-I } & \multirow{2}{*}{$\begin{array}{l}\text { Number of } \\
\text { paired visits }\end{array}$} & \multicolumn{4}{|c|}{ Change (follow-up vs. baseline) } & \multirow{2}{*}{$\begin{array}{c}\text { Effect size } \\
\text {-(Cohen's d) }\end{array}$} & \multicolumn{5}{|c|}{ ROC analysis } \\
\hline & & & & Mean & $\begin{array}{l}\text { Standard } \\
\text { Deviation }\end{array}$ & 95th C & & & $\begin{array}{l}\text { Optimal } \\
\text { cutoff }\end{array}$ & Sensitivity & Specificity & $\mathrm{LR}+$ & LR- \\
\hline \multirow[t]{3}{*}{$\begin{array}{l}\text { UDysRS Part I (On } \\
\text { Dyskinesia) }\end{array}$} & 3 & $\begin{array}{l}\text { a little } \\
\text { better }\end{array}$ & 248 & -2.1 & 3.9 & -3.7 & -1.5 & 0.20 & -2.5 & 0.562 & 0.644 & 1.601 & 0.699 \\
\hline & 4 & the same & 386 & -0.5 & 3.7 & -1.3 & 0.9 & 0.02 & NA & & & & \\
\hline & 5 & $\begin{array}{l}\text { a little } \\
\text { worse }\end{array}$ & 203 & 1.8 & 3.8 & 0.9 & 3.2 & 0.18 & 1.5 & 0.577 & 0.597 & 1297 & 0.802 \\
\hline \multirow[t]{3}{*}{$\begin{array}{l}\text { UDysRS Part II (OFF } \\
\text { Dystonia) }\end{array}$} & 3 & $\begin{array}{l}\text { a little } \\
\text { better }\end{array}$ & 248 & -1.8 & 2.7 & -2.9 & 1.5 & 0.21 & -1.5 & 0.654 & 0.701 & 1.718 & 0.621 \\
\hline & 4 & the same & 386 & 0.0 & 2.9 & -0.5 & 0.6 & 0.01 & NA & & & & \\
\hline & 5 & $\begin{array}{l}\text { a little } \\
\text { worse }\end{array}$ & 203 & 1.7 & 3.2 & 0.7 & 2.4 & 0.19 & 1.5 & 0.676 & 0.623 & 1.207 & 0.830 \\
\hline \multirow[t]{3}{*}{ MDS-UPDRS Part IV } & 3 & $\begin{array}{l}\text { a little } \\
\text { better }\end{array}$ & 248 & -0.9 & 3.5 & -1.4 & -0.4 & 0.18 & -0.5 & 0.524 & 0.634 & 1.402 & 0.724 \\
\hline & 4 & the same & 386 & $-0,2$ & 3.1 & -0.9 & 0.3 & 0.01 & NA & & & & \\
\hline & 5 & $\begin{array}{l}\text { a little } \\
\text { worse }\end{array}$ & 203 & 0.8 & 3.6 & 0.4 & 1.3 & 0.15 & 0.5 & 0.582 & 0.597 & 1251 & 0801 \\
\hline
\end{tabular}

The number of return visits did not appreciably alter these values.

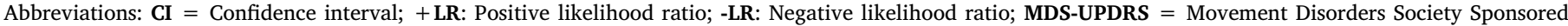

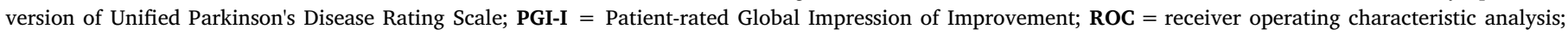
UDysRS = Unified Dyskinesia Rating Scale.

applicability of our calculations.

To the best of our knowledge, our study is the first establishing the MCID thresholds for the UDysRS Parts 1 and 2 and MDS-UPDRS MC. Therefore, we cannot compare our results to those of other groups.

In a PubMed search performed on February 15, 2018 (key words: "Unified Dyskinesia Rating Scale" and "Parkinson's disease"), we identified 6 studies reporting changes in UDysRS subscales as their outcomes.

In a randomized and controlled pilot trial, Fox et al. examined the effectiveness of the sigma-1 receptor-agonist and glutamatergic/ monoaminergic modulator, dextromethorphan, plus quinidine for treating levodopa-induced dyskinesia [24]. Their secondary endpoints included several dyskinesia/motor assessments. Although statistical significance was not reached (p values 0.057-0.407), the observed changes in MDS-UPDRS MC and UDysRS Part 1 were clinically important ( -2.7 and -4.0 , respectively) [24]. On the other hand, changes in UDysRS Part 2 were neither statistically, nor clinically, relevant.

Three different articles have been published on the efficacy of ADS5102 (Amantadine extended release) in PD with motor complications [25-27]. None of these studies reported the changes in the individual UDysRS subscales; only the composite scores (i.e., total score) were published. Except for the dose of $260 \mathrm{mg}$, the MDS-UPDRS MC scores improved in both statistically significant and clinically relevant manners. In their pooled analysis published recently [28], changes in the
MDS-UPDRS MC showed a clinically important treatment effect for ADS-5102 at week 12 [mean change: -2.3 , 95th confidence interval (CI): -3.2 and $-1.5, \mathrm{p}<0.0001]$.

Juhasz et al. evaluated the effects of LCIG on the motor complication in a prospective, multicenter and an open-label trial. A total of 34 subjects finished the study. All dyskinesia-related outcomes demonstrated statistically significant improvement (UDysRS Part 1 from $20.5 \pm 8.6$ to $14.5 \pm 9.3$ points, UDysRS Part 2 from $9.5 \pm 4.7$ to $6.3 \pm 4.2$ points, and MDS-UPDRS MC from $10.4 \pm 4.0$ to $7.5 \pm 4.0$ points, $\mathrm{p}<0.01$ in all cases), which also meet our criteria for clinical relevance [29].

In another prospective trial, Juhasz et al. examined the efficacy of bilateral subthalamic DBS therapy on dyskinesia. Of note, the changes in the UDysRS total score was the primary outcome. Based on the data of 71 consecutive patients, the authors showed an $11.7 \pm 8.4$ point and $5.7 \pm 5.0$ point decrease on UDysRS Parts 1 and 2 , whereas the MDS-UPDRS MC scores were lowered by $4.0 \pm 3.6$ points. Again, these results can be considered as both statistically and clinically relevant [30].

In conclusion, the UDysRS and MDS-UPDRS MC tests have been successfully applied in numerous interventional studies measuring the changes in dyskinesia. Our MCID estimations can provide guidance on judging the relevance of the results of the published and future trials from a clinical perspective.

Table 2

Minimal clinically important difference threshold values based on disease severity.

\begin{tabular}{|c|c|c|c|c|c|c|}
\hline Instrument & PGI- & & Mild (HYS 1\&2) & Moderate (HYS 3) & Severe (HYS $4 \& 5$ ) & Overall (HYS 1-5) \\
\hline UDysRS Part I (On Dyskinesia) & 5 & a little worse & 1.4 & 2.2 & 2.4 & 1.8 \\
\hline \multirow[t]{2}{*}{ UDysRS Part II (OFF Dystonia) } & 3 & a little better & -1.4 & -1.9 & -2.2 & -1.8 \\
\hline & 4 & the same & -0.1 & 0.1 & 0.2 & 0.0 \\
\hline \multirow{2}{*}{ MDS-UPDRS Part IV } & 4 & the same & 0.1 & -0.1 & -0.3 & $-0,2$ \\
\hline & 5 & a little worse & 1.1 & 0.7 & 1.0 & 0.8 \\
\hline
\end{tabular}

Abbreviations: HYS: Hoehn-Yahr Stage; MDS-UPDRS = Movement Disorders Society Sponsored version of Unified Parkinson's Disease Rating Scale; UDysRS $=$ Unified Dyskinesia Rating Scale. 


\section{Author roles}

1. Research project: A. Conception, B. Organization, C. Execution;

2. Statistical Analysis:A. Design, B. Execution, C. Review and Critique;

3. Manuscript: A. Writing of the first draft, B. Review and Critique.

AM 1, 2, 3.

DP $1 \mathrm{~B}, 2 \mathrm{C}, 3 \mathrm{~B}$.

MK 1B, 2C, 3B.

JJ 1A, 2C, 3B.

NK $1,2,3$.

\section{Financial disclosures}

AM reported no financial disclosure.

JJ received $<1000$ EUR consultation fees from Hungarian subsidiaries of UCB, Valeant and Eisai. Regarding this pilot study the author did not receive any corporate funding.

MK reported no financial disclosure.

DP reported no financial disclosure.

NK received $<1000$ EUR consultation fees from Hungarian subsidiaries of Medtronic. Boehringer Ingelheim. Novartis. GlaxoSmithKline. UCB, Krka and Abbvie. Regarding this study the author did not receive any corporate funding.

\section{Conflicts of interest}

None is declared. This study was supported by the Hungarian Brain Research Program (2017-1.2.1-NKP-2017-00002), NKFIH EFOP-3.6.216-2017-00008, NKFIH SNN125143 and ÚNKP-17- 4 -I.- PTE-311 government-based funds. Our research was partly financed by the Higher Education Institutional Excellence Program of the Ministry of Human Capacities in Hungary, within the framework of the 5th thematic program of the University of Pécs, Hungary (A 20765/3/2018/ FEKUSTRAT).

\section{Acknowledgments}

This study was supported by the Bolyai Scholarship of the Hungarian Academy of Sciences, Hungarian Brain Research Program (2017-1.2.1-NKP-2017-00002) and OTKA SNN125143 governmentbased funds. We would thank Jon Marquette for his language editing. Our research was partly financed by the Higher Education Institutional Excellence Program of the Ministry of Human Capacities in Hungary, within the framework of the 5th thematic program of the University of Pécs, Hungary (A 20765/3/2018/FEKUSTRAT).

\section{Appendix A. Supplementary data}

Supplementary data related to this article can be found at https:// doi.org/10.1016/j.parkreldis.2018.08.018.

\section{References}

[1] C.G. Goetz, J.G. Nutt, G.T. Stebbins, The unified dyskinesia rating scale: presentation and clinimetric profile, Mov. Disord. 23 (16) (2008) 2398-2403.

[2] C.G. Goetz, G.T. Stebbins, L. Wang, N.R. LaPelle, S. Luo, B.C. Tilley, IPMDS-sponsored scale translation program: process, format, and clinimetric testing plan for the MDS-UPDRS and UDysRS, Mov. Disord. Clin. Pract. 1 (2) (2014) 97-101.

[3] S. Luo, Y. Liu, J.A. Teresi, G.T. Stebbins, C.G. Goetz, Differential item functioning in the unified dyskinesia rating scale (UDysRS), Mov. Disord. 32 (8) (2017)
1244-1249.

[4] C.G. Goetz, G.T. Stebbins, A. Theeuwes, et al., Temporal stability of the unified dyskinesia rating scale, Mov. Disord. 26 (14) (2011) 2556-2559.

[5] C.G. Goetz, G.T. Stebbins, K.A. Chung, et al., Which dyskinesia scale best detects treatment response? Mov. Disord. 28 (3) (2013) 341-346.

[6] E. Cubo, J.M. Gabriel-Galan, J.S. Martinez, et al., Comparison of office-based versus home Web-based clinical assessments for Parkinson's disease, Mov. Disord. 27 (2) (2012) 308-311.

[7] E. Cubo, C.G. Goetz, G.T. Stebbins, et al., Independent Spanish validation of the unified dyskinesia rating scale, Mov. Disord. Clin. Pract. 1 (3) (2014) 213-218.

[8] K. Horvath, Z. Aschermann, P. Acs, et al., Az Egységesített dyskinesia Pontozóskála magyar nyelvi validációja (Validation of the Hungarian unified dyskinesia rating scale), Ideggyogyaszati Szle. 68 (5-6) (2015) 183-188.

[9] M. Skorvanek, M. Minar, M. Grofik, et al., Validation of the official Slovak version of the unified dyskinesia rating scale (UDysRS), Parkinsons Dis. 2015 (2015) 674796.

[10] C.G. Goetz, B.C. Tilley, S.R. Shaftman, et al., Movement disorder society-sponsored revision of the unified Parkinson's disease rating scale (MDS-UPDRS): scale presentation and clinimetric testing results, Mov. Disord. 23 (15) (2008) 2129-2170.

[11] T.A. Mestre, I. Beaulieu-Boire, C.C. Aquino, et al., What is a clinically important change in the Unified Dyskinesia Rating Scale in Parkinson's disease? Park. Relat. Disord. 21 (11) (2015) 1349-1354.

[12] K. Horvath, Z. Aschermann, M. Kovacs, et al., Minimal clinically important differences for the experiences of daily living parts of movement disorder society-sponsored unified Parkinson's disease rating scale, Mov. Disord. 32 (5) (2017) 789-793.

[13] K. Horvath, Z. Aschermann, P. Acs, et al., Minimal clinically important difference on the Motor Examination part of MDS-UPDRS, Park. Relat. Disord. 21 (12) (2015) 1421-1426.

[14] A. Makkos, M. Kovacs, Z. Aschermann, et al., Are the MDS-UPDRS-based composite scores clinically applicable? Mov. Disord. 33 (5) (2018 May) 835-839, https://doi. org $/ 10.1002 /$ mds. 27303.

[15] K. Horváth, Z. Aschermann, P. Ács, et al., Validation of the Hungarian MDS-UPDRS: why do we need a new Parkinson scale? Ideggyogyaszati Szle. 67 (3-4) (2014) 129-134.

[16] R.A. Hauser, M.F. Gordon, Y. Mizuno, et al., Minimal clinically important difference in Parkinson's disease as assessed in pivotal trials of pramipexole extended release, Parkinsons Dis 2014 (2014) 467131.

[17] T. Lucza, K. Karadi, J. Kallai, et al., Screening mild and major neurocognitive disorders in Parkinson's disease, Behav. Neurol. 2015 (2015) 983606.

[18] T. Lucza, K. Karadi, S. Komoly, et al., Neurocognitive disorders in Parkinson's disease, Orv. Hetil. 156 (23) (2015) 915-926.

[19] R. Weintraut, K. Karádi, T. Lucza, et al., Lille apathy rating scale and MDS-UPDRS for screening apathy in Parkinson's disease, J. Parkinson's Dis. 6 (1) (2016) $257-265$.

[20] D. Revicki, R.D. Hays, D. Cella, J. Sloan, Recommended methods for determining responsiveness and minimally important differences for patient-reported outcomes, J. Clin. Epidemiol. 61 (2) (2008) 102-109.

[21] R. Jaeschke, J. Singer, G.H. Guyatt, Measurement of health status. Ascertaining the minimal clinically important difference, Contr. Clin. Trials 10 (4) (1989) 407-415.

[22] J. Cohen, Statistical Power Analysis for the Behavioral Sciences, second ed., Lawrence Earlbaum Associates, Hillsdale, NJ, 1988.

[23] G. Samsa, D. Edelman, M.L. Rothman, G.R. Williams, J. Lipscomb, D. Matchar, Determining clinically important differences in health status measures: a general approach with illustration to the Health Utilities Index Mark II Pharmacoeconomics 15 (2) (1999) 141-155.

[24] S.H. Fox, L.V. Metman, J.G. Nutt, et al., Trial of dextromethorphan/quinidine to treat levodopa-induced dyskinesia in Parkinson's disease, Mov. Disord. 32 (6) (2017) 893-903.

[25] W. Oertel, K. Eggert, R. Pahwa, et al., Randomized, placebo-controlled trial of ADS5102 (amantadine) extended-release capsules for levodopa-induced dyskinesia in Parkinson's disease (EASE LID 3), Mov. Disord. 32 (12) (2017) 1701-1709.

[26] R. Pahwa, C.M. Tanner, R.A. Hauser, et al., ADS-5102 (amantadine) extended-release capsules for levodopa-induced dyskinesia in Parkinson disease (EASE LID study): a randomized clinical trial, JAMA Neurol. 74 (8) (2017) 941-949.

[27] R. Pahwa, C.M. Tanner, R.A. Hauser, et al., Amantadine extended release for levodopa-induced dyskinesia in Parkinson's disease (EASED Study), Mov. Disord. 30 (6) (2015) 788-795.

[28] L.W. Elmer, J.L. Juncos, C. Singer, et al., Pooled analyses of phase III studies of ADS5102 (Amantadine) extended-release capsules for dyskinesia in Parkinson's disease, CNS Drugs 32 (4) (2018 Apr) 387-398, https://doi.org/10.1007/s40263-0180498-4.

[29] A. Juhasz, Z. Aschermann, P. Acs, et al., Levodopa/carbidopa intestinal gel can improve both motor and non-motor experiences of daily living in Parkinson's disease: an open-label study, Park. Relat. Disord. 37 (2017) 79-86.

[30] A. Juhasz, G. Deli, Z. Aschermann, et al., How efficient is subthalamic deep brain stimulation in reducing dyskinesia in Parkinson's disease? Eur. Neurol. 77 (5-6) (2017) 281-287. 